\title{
Global networks of small telescopes will chase companion signals of gravitational waves
}

Seeing cosmic events is one thing, but what if you could hear them and taste them, too?

\author{
Davide Castelvecchi
}

13 October 2017 Clarified: 01 November 2017

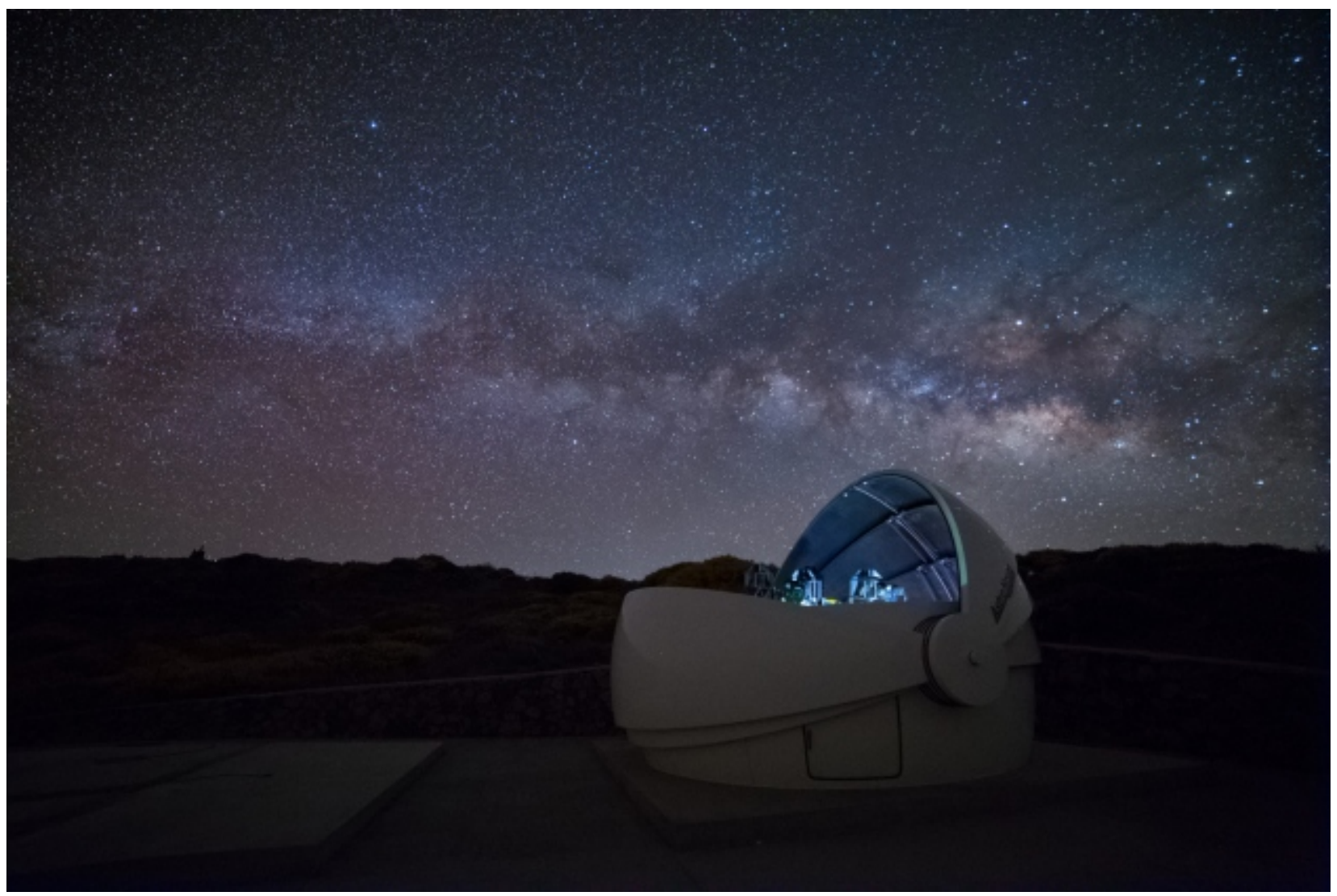

Krzysztof Ulaczyk/University of Warwick

The Gravitational-wave Optical Transient Observer (GOTO) in La Palma, Spain, will look for flares of light coming from the same spot as any gravitational waves.

A cottage industry of small observatories is springing up around the globe to take advantage of astronomers' new ability to capture the gravitational waves from major cosmic events. These new facilities will enable researchers to match up those gravitational waves with electromagnetic signals and perhaps one day even particles of matter from some of the cataclysms that send measurable ripples through space-time. 
The main goal is to look for flares of light originating from the same spot as any gravitational waves detected by the US-based Advanced Laser Interferometer Gravitational-Wave Observatory (LIGO), or the Virgo observatory near Pisa, Italy. These smaller telescopes, often built on a shoestring budget, will serve as first-line responders, filling the gap between gravitational-wave detectors and the major facilities of conventional astronomy. "Once you know where to look, you can swing the whole world's telescopes at it," says Danny Steeghs, an astronomer at the University of Warwick, UK.

Moving quickly is key. It's tricky to pinpoint the source of gravitational waves — astronomers can typically narrow it down to a region of the Universe that could contain thousands of galaxies - and observatories may have only a few days before any promising flares of light dissipate. "You need to look at a lot of sky," says Steeghs, "and you don't have a lot of time for it."

\section{Robots of the sky}

Steeghs leads a small

UK-Australian collaboration that built the Gravitational wave Optical Transient Observer (GOTO) in La Palma, Spain. It is an array of four small robotic telescopes that will eventually grow to 8 telescopes, and perhaps 16. So far, it has cost just $£ 800,000$ (around US\$1 million).

\section{Related stories}

- European detector spots its first gravitational wave

- Gravitational waves: 6 cosmic questions they can tackle

- Global observatory sees first light

\section{Related stories}

- European detector spots its first gravitational wave

- Gravitational waves: 6 cosmic questions they can tackle

- Global observatory sees first light

Alan Watson and William Lee of the

National Autonomous University of Mexico (UNAM) in Mexico City and his collaborators spent even less. They built the Deca-Degree Optical Transient Imager (DDOTI), currently consisting of a pair of robotic telescopes at Sierra San Pedro Martir, Mexico, for a mere US $\$ 350,000$, largely by using off-theshelf components, he says. They plan eventually to have six telescopes, perhaps followed by more facilities in France and Australia.

Some of the facilities, including GOTO, are being designed and built specifically to follow up on gravitational-wave signals. Most of these will be robotic, using machine-learning algorithms to alert each other to point at particular regions of sky and search for interesting flares without the need for human intervention. 
Other projects have grown out of existing collaborations that are familiar with looking for visible-light counterparts to the $y$-ray bursts spotted by space observatories, or tracking other transient phenomena, such as supernovae explosions or asteroids that are potentially Earth-bound. And some venerable telescopes, including one of those once used by Edwin Hubble in Palomar, California, have been retrofitted. The 1.2-metre telescope is now part of GROWTH (Global Relay of Observatories Watching Transients Happen), a network of 17 facilities around the globe that can track an object seamlessly as the Earth spins. "The idea is, basically, to beat sunrise," says Mansi Kasliwal, an astronomer at the California Institute of Technology in Pasadena, who leads GROWTH.

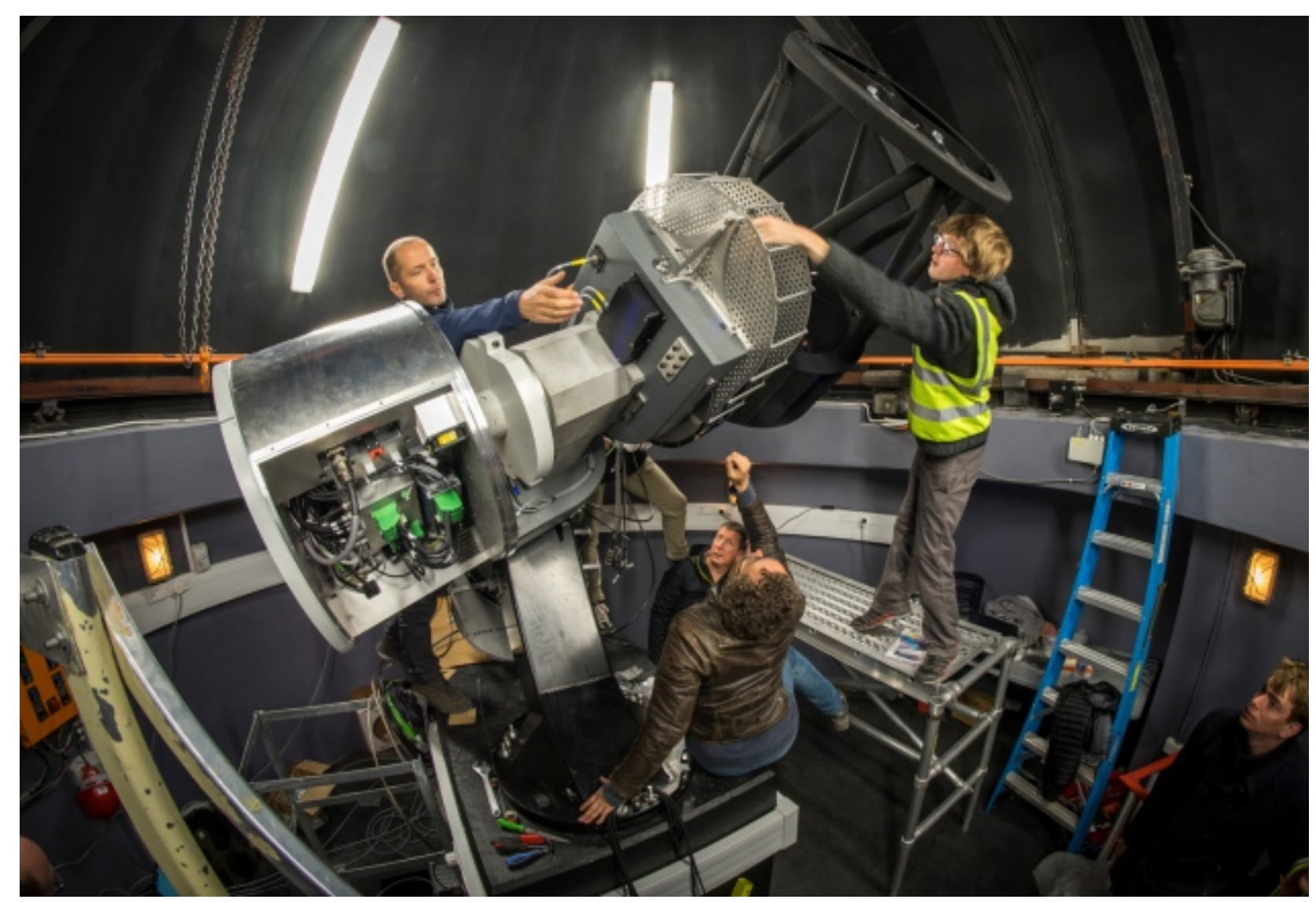

Twan Bekkers

Engineers install a prototype of the BlackGEM telescopes at the South African Astronomical Observatory in Sutherland.

Astrophysicist Paul Groot of Radboud University in Nijmegen, the Netherlands, whose group is part of the Virgo collaboration itself, is leading a Dutch-funded project called BlackGEM. It will initially consist of three telescopes in La Silla, Chile, costing about $€ 6$ million (US\$7.1 million), that will continuously map the southern sky to build up a database of archived images. If news of a gravitational-wave 
detection arrives, BlackGEM will scan the relevant patch of sky within hours, and automatically compare that to its archived images to search for anything new.

\section{Neutrino chasers}

Similar efforts are already following up on detections of notable particles from space, such as unusually energetic neutrinos or cosmic rays. The Astrophysical Multimessenger Observatory Network (AMON), started in 2016, got its first interesting hint on 22 September, when it responded to a highenergy neutrino detected by IceCube, the world's largest neutrino observatory, at the South Pole.

When AMON researchers looked towards the source of the neutrino, they saw that a known quasar an entity consisting of heated matter orbiting a supermassive black hole at the centre of a distant galaxy - was flaring up. This is the type of heightened activity that theorists think could produce an excess of neutrinos, but so far, no high-energy neutrinos have been traced conclusively back to their sources.

In the future, researchers hope that they might detect all three types of emission together: electromagnetic radiation, gravitational waves and particles of matter. Some compare that to seeing, hearing and tasting an astrophysical event at once.

Nature doi: $10.1038 /$ nature.2017.22828

\section{Clarifications}

Clarified: A previous version of this article misstated the roles of two people. Mansi Kasliwal is not just a part of GROWTH, she leads it, and Alan Watson shares the leadership role of DDOTI with William Lee. 